\title{
Human Cells, Tissues, and Cellular and Tissue-Based Products Processing
}

National Cancer Institute

\section{Source}

National Cancer Institute. Human Cells, Tissues, and Cellular and Tissue-Based Products

Processing. NCl Thesaurus. Code C133285.

Activities performed on Human Cells, T issues, and Cellular and Tissue-Based Products

(HCT/Ps) other than recovery, donor screening and testing, storage, labeling, packaging or distribution. 